\title{
Position paper on requirements for toxicological studies in the specific case of radiopharmaceuticals
}

\author{
J. Koziorowski ${ }^{*}$, M. Behe ${ }^{2}$, C. Decristoforo ${ }^{3}$, J. Ballinger ${ }^{4}$, P. Elsinga ${ }^{5}$, V. Ferrari ${ }^{6}$, P. Kolenc Peitl', S. Todde ${ }^{8}$ \\ and T. L. Mindt ${ }^{*}$
}

* Correspondence:

jacek.koziorowski@regionostergotland. se; t.mindt@gmx.ch

${ }^{1}$ Department of Radiation Physics and Department of Medical and Health Sciences, Linköping

University, Linköping, Sweden ${ }^{9}$ University of Basel Hospital, Radiopharmaceutical Chemistry, 4031 Basel, Switzerland

Full list of author information is available at the end of the article

\section{Springer}

\begin{abstract}
This is a position paper of the Radiopharmacy Committee of the EANM (European Association of Nuclear Medicine) addressing toxicology studies for application of new diagnostic and therapeutic radiopharmaceuticals (RP) that are not approved (i.e., not having a marketing authorization or a monograph in the European Pharmacopoeia), excluding endogenous and ubiquitous substances in humans. This paper discusses the requirements for clinical trials with radiopharmaceuticals for clinical research applications, not necessarily intended to aim at a marketing authorization. If marketing authorization is intended, scientific advice of the competent authorities is mandatory and cannot be replaced by this position paper. The position paper reflects the view of the Radiopharmacy Committee of the EANM and can be used as a basis for discussions with the responsible authorities.
\end{abstract}

Keywords: Position paper, Pre-clinical toxicology studies, Radiopharmaceuticals

\section{Background}

The existing guideline on toxicology studies applicable to RP "ICH guideline M3(R2) on non-clinical safety studies for the conduct of human clinical trials and marketing authorisation for pharmaceuticals" (CPMP/ICH/286/95) leaves some room for interpretation and is not always clear in describing the type of toxicology study required for various RP applications. Our intention is to give guidance for the specific case of RP and to include other guidelines on impurities in new drug substances "Note for guidance on impurity testing in new drug substances" (CPMP/ICH/2737/99) and genotoxic impurities "Guideline on the limits for genotoxic impurities" (CPMP/SWP/5199/02), "Opinion on use of the threshold of toxicological concern (TTC) approach for human safety assessment of chemical substances with focus on cosmetics and consumer products" (SCCP/1171/08) and FDA's "Guidance for industry, Developing medical imaging drugs and biological products, Part 1: conducting safety assessments" and FDA's "Guidance for Industry, Investigators and Reviewers, Exploratory IND studies". In another important document, "ICH guideline S9 on the nonclinical evaluation of anticancer pharmaceuticals" (CHMP/ICH/646107/08) radiopharmaceuticals are not specifically mentioned, even though oncology is a major field of application for RPs.

(c) 2016 Koziorowski et al. Open Access This article is distributed under the terms of the Creative Commons Attribution 4.0 International License (http://creativecommons.org/licenses/by/4.0/), which permits unrestricted use, distribution, and reproduction in any medium, provided you give appropriate credit to the original author(s) and the source, provide a link to the Creative Commons license, and indicate if changes were made. 


\section{Considerations on preclinical toxicity studies for radiopharmaceuticals}

Acute toxicity studies: These are intended to determine the risk of overdosage. Mortality, toxic symptoms and lesions observed during autopsy are registered to determine the no observed effect level (NOEL). This type of study was "traditionally" used as main parameter for toxicity determination before human application of radiopharmaceuticals. Today, it is mainly replaced by so-called extended single dose toxicity studies.

Some type of studies are typically not performed for radiopharmaceuticals these include:

- Subacute and chronic toxicological studies (RP exposure is limited to a single dose or a few doses only)

- Teratogenicity (reproduction) studies (RPs are not given to pregnant women)

- Genotoxic (mutagenic) studies (exposure is limited to a single dose or a few doses only. In certain cases desktop screening (in silico) or the Ames test are sufficient)

- Carcinogenic studies (radiation is a major contributor to cancer induction and this is covered by dosimetric considerations).

For biotechnology-derived products, appropriate nonclinical safety studies should be conducted in accordance with the safety guideline ICH S6 [ICH guideline S6 (R1) - preclinical safety evaluation of biotechnology-derived pharmaceuticals, EMA/CHMP/ICH/731268/1998, European Medicines Agency]. The "Draft guideline on immunogenicity assessment of biotechnology-derived therapeutic proteins" (EMEA/CHMP/BMWP/14327/2006 Rev. 1) may also be applicable.

\section{Considerations on the compound to be tested for toxicity}

Radiopharmaceuticals are prepared by the reaction of a radionuclide with a nonradioactive precursor. Due to the short half-life of most radionuclides employed the preparation is typically done onsite immediately before clinical application. This step is generally performed on the basis of chemical reactions which are adapted in such a way as to meet appropriate radiochemical conditions. There are two basic scenarios:

A) The radiolabelling is characterized by a quantitative reaction of a radionuclide with a chemical precursor, which does not require a purification step. Typically, the precursor is used in large molar excess over the radionuclide (e.g., a complexation agent/ligand over a radiometal). All components, including the precursor (or, e.g., precursor hydrolysis product) and the resulting radiopharmaceutical active ingredient are co-injected.

In this case, the precursor (or e.g., precursor hydrolysis product) should be used for preclinical toxicity studies. A risk assessment should describe the lowest potential excess of precursor over radionuclide. In the case of doubt, preclinical data comparing the pharmacological activity (e.g., affinity to a receptor) of the precursor and the radiolabelled compound should be provided to support this approach.

B) In cases where radiolabelling reactions are not quantitative, an efficient purification is required to separate the desired radioactive compound from the reaction mixture and ensure low levels of radionuclidic, chemical and/or radiochemical impurities. This involves isolation of the radiopharmaceutical active ingredient, typically by 
HPLC, and subsequent formulation to the radiopharmaceutical preparation to be injected.

In these cases the molecule containing a stable instead of a radioactive isotope should be used (e.g. ${ }^{19} \mathrm{~F}$ instead of ${ }^{18} \mathrm{~F}$ ) for toxicity testing. Data should be provided to show the sufficient separation process of precursor and radiopharmaceutical active ingredient including the mass amount of precursor and radiopharmaceutical active ingredient in the final formulation.

\section{Proposal: toxicology limits for radiopharmaceuticals}

Based on the above outlined considerations and existing guidelines, a new approach for toxicology assessment based upon the definition of three distinct toxicological limits is proposed: 1) $<1.5 \mu \mathrm{g}, 2)<100 \mu \mathrm{g}$ and 3) $>100 \mu \mathrm{g}$.

\section{$<1.5 \mu \mathrm{g}$}

This limit is based on the Threshold of Toxicological Concern (TTC) concept. A TTC value of $1.5 \mu \mathrm{g} /$ day intake of a genotoxic impurity is considered to be associated with an acceptable risk (excess cancer risk of $<1$ in 100,000 over a lifetime) for most pharmaceuticals. It should be noted that in "Note for guidance on impurities in new drug products" (CPMP/ICH/2738/99) a total daily intake (TDI) of an impurity may be up to $2 \mathrm{mg}$.

Based on case-by-case judgment for RPs applied in amounts of $<1,5 \mu \mathrm{g}$ injected dose, no toxicology tests may be required, however potential toxicity should be addressed and discussed. The evaluation of potential toxicity may be performed by desktop screening (in silico) and (quantitative) structure-activity relationship (Q)SAR). FDA is considering a tiered approach which ranges from $<1,5 \mu \mathrm{g} /$ person/day as a general threshold, $<15 \mu \mathrm{g} /$ person/day for chemicals without structural alerts for carcinogenicity or with negative mutagenicity test results (Ames test) and $<45 \mu \mathrm{g} /$ person/day for chemicals without structural alerts for carcinogenicity or with negative mutagenicity test results (Ames test) and a LD50 (median lethal dose) $>1000 \mathrm{mg} / \mathrm{kg}$ bodyweight.

Compounds excluded from this concept: high potency genotoxic carcinogens such as aflatoxin-like-, N -nitroso-, and azoxy-compounds. Risk assessment of members of such groups requires compound-specific toxicity data [Guideline on the limits for genotoxic impurities, CPMP/SWP/5199/02, European Medicines Agency; Opinion on Use of the Threshold of Toxicological Concern (TTC) Approach for Human Safety Assessment of Chemical Substances with focus on Cosmetics and Consumer Products, SCCP/1171/08, European Commission; ICH guideline M7 on assessment and control of DNA reactive (mutagenic) impurities in pharmaceuticals to limit potential carcinogenic risk, EMA/ CHMP/ICH/83812/2013, European Medicines Agency]. Any substance with positive Ames test should be evaluated on a case-by-case basis. It must be mentioned that the TTC approach allows up to $120 \mu \mathrm{g}$ for a single dose in a recent ICH document (ICH M7) soon replacing current guidelines that suggests even room for extension of this concept [Multidisciplinary ICH guidelines including M 7].

$<100 \mu \mathrm{g}$

In this case (so-called microdosing) the "Note for guidance on non-clinical safety studies for the conduct of human clinical trials and marketing authorization for pharmaceuticals" (CPMP/ICH/286/95) can be applied. 
For <100 $\mu$ g, approach 1 in "ICH guideline M3(R2) on non-clinical safety studies for the conduct of human clinical trials and marketing authorisation for pharmaceuticals" is applicable. Typically, a $100 \mathrm{x}$ times the clinical dose in thirty animals (rodents) and examination in ten animals/sex on the day following the injection and five animals/sex after 14 days (hematology, clinical chemistry, necropsy, and histopathology) should be used. The in vivo toxicology tests must be performed in compliance with Good Laboratory Practice (GLP), interspecies scaling (allometric scaling) should be applied to calculate from animal to human dose [Note for guidance on non-clinical safety studies for the conduct of human clinical trials and marketing authorization for pharmaceuticals, CPMP/ICH/286/95, European Medicines Agency].

The $1000 \mathrm{x}$ time scaling is mentioned in the same guideline approach and may be followed if the allometric scaling is not used. The exact approach should be negotiated with the competent authorities.

The $100 \mathrm{x}$ allometric scaling is also in agreement with FDA's "Guidance for industry, Developing medical imaging drugs and biological products, Part 1: conducting safety assessments":

"We recommend that the NOAEL in safety pharmacology studies in suitable animal species be at least one hundred times (100x) greater than the maximal mass dose to be used in human studies."

It is recommended for all approaches to use a body weight of $50 \mathrm{~kg}$ for a patient to be on the safe side.

A major limitation of this microdosing approach is that it does not take into account that pharmacological and toxicological effects are usually not determined by the mass but the molar amount to be applied. For larger molecules such as peptides or proteins this causes a major limitation of the microdosing concept based solely on the mass. It should be noted, that the average molecular weight $\left(\mathrm{M}_{\mathrm{w}}\right)$ of a drug compound is approx. $300 \mathrm{~g} / \mathrm{mol}$.

Therefore, from a pharmacological point of view a peptide with a $M_{w}$ of approx. $1500 \mathrm{~g} / \mathrm{mol}$ having the same pharmacological potency as a low molecular weight drug could be used in a five-fold higher amount. In such a case the microdosing concept could be extended to, e.g., $<500 \mu \mathrm{g}$ and still be within the same equimolar limit. For larger molecules such as proteins the FDA's "Guidance for Industry, Investigators, and Reviewers; Exploratory IND Studies" sets the limit to $<30$ nmoles: "Due to differences in molecular weights as compared to synthetic drugs, the maximum dose for protein products is $\leq 30$ nanomoles." This corresponds to $<100 \mu \mathrm{g}$ of a drug having a molecular weight of $300 \mathrm{~g} / \mathrm{mol}$.

It also should be noted that for radiopharmaceuticals, usually extensive biodistribution data (often including imaging) from preclinical studies are available. These studies give detailed quantitative data on accumulation and elimination in tissues and excretion pathways. Based on such data the design of extended single dose toxicity studies may be focused on risk organs and tissues, thereby eliminating the requirement especially for (high cost) histopathological data in all organs, focusing on main organs where the RP accumulates by, e.g., binding to receptors or other target structures. Such design has to be made on a case-by case basis and its rational being described in detail in the application process.

\section{$>100 \mu \mathrm{g}$}

Applications of more than $100 \mu \mathrm{g}$ substance in the case of RPs may be required for imaging with peptides and proteins or for therapeutic applications. The assumptions made 
focus on single dose applications in clinical studies, i.e., the RP is expected to be cleared completely from the blood before the next application.

\section{For imaging}

In this case, also the "Note for guidance on non-clinical safety studies for the conduct of human clinical trials and marketing authorization for pharmaceuticals" can be applied using approach 3. This includes also an extended single dose toxicity study, however, in this case in rodent and non-rodent species, as well as a test for genetoxicity (usually Ames test). Selection of histopathology data may be based on the same considerations as described in the $<100 \mu \mathrm{g}$ group. The toxicology tests must be performed in compliance with Good Laboratory Practice (GLP).

\section{For therapy}

For the anticancer use of RPs (immunoradiotherapy, endoradiotherapy) the "ICH guideline S9 on nonclinical evaluation for anticancer pharmaceuticals" can be employed. In these cases in particular genetoxicity testing for Phase I and II studies may not be required.

In accordance with scenario in section $<100 \mu \mathrm{g}$, the argument of molar equivalence may be used.

\section{Conclusion}

The concept of differentiating 3 toxicological limits will clarify the requirements for application of clinical trials of new radiopharmaceuticals and allow much better planning of translational studies, thereby strengthening research efforts towards novel targeting agents for molecular imaging and molecular radiotherapy. It is our aim to have clear recommendations from competent authorities, such as EMA, for the requirements on toxicological studies for radiopharmaceuticals. In the future we recommend that the mass limits will be replaced by molar amounts as this makes more sense from a pharmacological perspective and would facilitate (radio)pharmaceutical development and practice.

The guidelines give some general recommendations for necessary toxicological studies. But it is necessary to make a risk assessment for each compound to evaluate which toxicological studies are needed and useful. Therefore a scientific advice meeting with the competent authorities would be very helpful before starting expensive toxicity studies.

\section{Abbreviations}

(Q)SAR: (Quantitative) structure-activity relationship; API: Active pharmaceutical ingredient; CHMP: Committee for Medicinal Products for Human Use; CPMP: Committee for proprietary medicinal products; EMA: European Medicines Agency; GLP: Good laboratory practice; ICH: International Conference on Harmonisation of Technical Requirements for Registration of Pharmaceuticals for Human Use; MTD: Maximum tolerated dose; NOAEL: No observed adverse effect level; NOEL: No observed effect level; PET: Positron emission tomography; SCCP: Scientific committee on consumer protection; SWP: Safety working party; TTC: Toxicological threshold of concern.

Competing interests

The authors declare that they have no competing interests.

\section{Authors' contributions}

$J K, M B, P E$, and TLM designed the study, analysed and interpreted the data, and drafted the manuscript. CD, JB, VF, PKP, and ST were involved in drafting the manuscript and revising it critically for content. All authors read and approved the final manuscript.

\section{Author details}

${ }^{1}$ Department of Radiation Physics and Department of Medical and Health Sciences, Linköping University, Linköping, Sweden. ${ }^{2}$ Center for Radiopharmaceutical Sciences ETH-PSI-USZ Paul-Scherrer-Institute, 5232 Villigen-PSI, Switzerland. ${ }^{3}$ Department of Nuclear Medicine, Medical University Innsbruck, 6020 Innsbruck, Austria. ${ }^{4}$ Guy's and St Thomas' 
Hospital, London SE1 9RT, UK. ${ }^{5}$ University Medical Center, University of Groningen, 9700 RB Groningen, Netherlands. ${ }^{6}$ GE Healthcare, Amersham, UK. ${ }^{7}$ University Medical Centre Ljubljana, 1000 Ljubljana, Slovenia. ${ }^{8}$ Università di Milano-Bicocca, Tecnomed Foundation, 20090 Monza, Italy. ${ }^{9}$ University of Basel Hospital, Radiopharmaceutical Chemistry, 4031 Basel, Switzerland.

Received: 7 November 2015 Accepted: 11 February 2016 1. 10.010

\section{References}

Guidance for industry, Developing medical imaging drugs and biological products, Part 1: conducting safety assessments, US Food and Drug Administration, http://www.fda.gov/downloads/Drugs/.../Guidances/ucm071600.pdf.

Guidance for Industry, Investigators and Reviewers, Exploratory IND studies, US Food and Drug Administration, http://www.fda.gov/downloads/drugs/guidancecomplianceregulatoryinformation/guidances/ucm078933.pdf.

Guideline on Immunogenicity assessment of biotechnology-derived therapeutic proteins, EMEA/CHMP/BMWP/14327/ 2006 Rev. 1, European Medicines Agency, http://www.ema.europa.eu/ema/doc_index.jsp?curl=pages/includes/ document/document_detail.jsp?webContentld=WC500194507\&murl=menus/document_library/document_library. jsp\&mid=0b01ac058009a3dc

Guideline on the limits for genotoxic impurities, CPMP/SWP/5199/02, European Medicines Agency, http://www.ema. europa.eu/docs/en_GB/document_library/Scientific_quideline/2009/09/WC500002903.pdf.

$\mathrm{ICH}$ guideline $\mathrm{M7}$ on assessment and control of DNA reactive (mutagenic) impurities in pharmaceuticals to limit potential carcinogenic risk, EMA/CHMP/ICH/83812/2013, European Medicines Agency, http://www.ema.europa.eu/ docs/en_GB/document_library/Scientific_guideline/2014/09/WC500173445.pdf.

ICH guideline S6 (R1) - preclinical safety evaluation of biotechnology-derived pharmaceuticals, EMA/CHMP/ICH/ 731268/1998, European Medicines Agency, http://www.ema.europa.eu/docs/en_GB/document_library/Scientific guideline/2009/09/WC500002828.pdf.

$\mathrm{ICH}$ guideline S9 on nonclinical evaluation for anticancer pharmaceuticals, CHMP/ICH/646107/08, European Medicines Agency, http://www.ema.europa.eu/docs/en_GB/document_library/Scientific_guideline/2010/01/WC500043471.pdf

Multidisciplinary ICH guidelines including M 7 can be found under http://www.ich.org/products/guidelines/ multidisciplinary/article/multidisciplinary-guidelines.html.

Note for guidance on impurities in new drug products, CPMP/ICH/2738/99, European Medicines Agency, http://www.ema.europa.eu/docs/en_GB/document_library/Scientific_guideline/2009/09/WC500002676.pdf.

Note for guidance on impurity testing in new drug substances, CPMP/ICH/2737/99, European Medicines Agency, http://www.ema.europa.eu/docs/en_GB/document_library/Scientific guideline/2009/09/WC500002675.pdf.

Note for guidance on non-clinical safety studies for the conduct of human clinical trials and marketing authorization for pharmaceuticals, CPMP/ICH/286/95, European Medicines Agency, http://www.ema.europa.eu/docs/en_GB/ document_library/Scientific_guideline/2009/09/WC500002941.pdf.

Opinion on use of the threshold of toxicological concern (TTC) approach for human safety assessment of chemical substances with focus on cosmetics and consumer products, SCCP/1171/08, European Commission, http://ec.europa.eu/health/scientific_committees/consumer_safety/docs/sccs_0_092.pdf.

\section{Submit your manuscript to a SpringerOpen ${ }^{\circ}$ journal and benefit from:}

- Convenient online submission

- Rigorous peer review

- Immediate publication on acceptance

- Open access: articles freely available online

- High visibility within the field

- Retaining the copyright to your article 